\title{
THE NON-EXPLOSIVE MODES OF HYDROGEN BURNING : A BRIEF REVIEW
}

\author{
MARCEL ARNOULD AND NAMI MOWLAVI \\ Institut d'Astronomie et d'Astrophysique \\ Université Libre de Bruxelles, C.P. 165; Av. F.D. Roosevelt, 50 \\ 1050 Bruxelles, BELGIUM
}

\begin{abstract}
The present status of our theoretical and experimental knowledge of the rates of hydrogen-burning reactions in hydrostatic stellar conditions is very briefly reviewed. Special emphasis is put on reactions of the proton-proton chain, $\mathrm{CNO}$ cycles, $\mathrm{NeNa}$ cycle and $\mathrm{MgAl}$ chain whose rates are still affected by uncertainties that can have an impact on predictions regarding astrophysical issues of interest. This concerns in particular the solar neutrino problem, or the nucleosynthesis accompanying the $\mathrm{CNO}, \mathrm{NeNa}$ and $\mathrm{MgAl}$ chains of reactions, as it is revealed in particular by the first dredge-up episode. A brief discussion of the possibility of reducing the present rate uncertainties is presented.
\end{abstract}

\section{INTRODUCTION}

Throughout their lives, stars transform nuclear and gravitational energy into photons and neutrinos. Such a transformation takes place through a succession of "controlled" thermonuclear burning stages interspersed with phases of gravitational contraction. The latter stages are responsible for a temperature increase, while the former ones produce nuclear energy and lead to composition changes.

The first major nuclear burning phase able to lead to the "complete" (hydrostatic plus thermal) equilibrium of a star is the combustion of hydrogen in its central regions, settling the object on the main sequence of the Hertzsprung-Russell diagram. After its exhaustion in the stellar core, hydrogen continues to burn in peripheral layers at later evolutionary stages.

Since the pioneering works of von Weizsäcker $(1937,1938)$, Bethe and Critchfield (1938) and Bethe (1939), it has become clear that those central and peripheral burning episodes take place through the "cold" p-p chains or CNO cycles, the dominant reaction sequence depending upon the stellar mass and initial composition (e.g. Rolfs and Rodney 1988, hereafter RR88). Other hydrogen burning modes, the "cold" $\mathrm{NeNa}$ and $\mathrm{MgAl}$ reaction chains have been identified more recently. They play a minor role in the stellar energy budget. In contrast, they are of importance in the synthesis of the $\mathrm{Na}$ to $\mathrm{Al}$ isotopes, especially in massive stars (e.g. RR88; Prantzos et al. 1986).

Much theoretical and laboratory effort has been devoted recently to an improvement of our knowledge of many of the nuclear reactions involved in 
the cold H-burning modes. In that field, experiments face enormous problems and represent a real challenge. Many of these difficulties relate to the fact that the energies of astrophysical interest for the charged particle induced reactions involved in the $H$-burning reactions mentioned above are much lower than the Coulomb barrier energies. As a consequence, the corresponding cross sections can dive into the nanobarn to picobarn abysses. In general, it has not been possible yet to measure directly such small cross sections. Theoreticians are thus requested to supply reliable extrapolations from the lowest energies attained experimentally to those of astrophysical relevance.

Progress in our knowledge of the rates of $\mathrm{H}$-burning reactions is indispensable in order to put various astrophysical predictions on safer grounds, like the solar neutrino flux, or the surface composition of low- or intermediate-mass Red Giant or Asymptotic Giant Branch stars having possibly experienced various dredge-up episodes able to pollute the outer convective zone with the products of central or shell H-burning (e.g. Sneden 1991 ; Iben 1991 for reviews). If freed from nuclear physics uncertainties to the largest possible extent, those calculated surface abundances could provide key constraints on the stellar structure from the main sequence to the Giant phase.

The aim of this contribution is to present a (non exhaustive) review of some recent experimental and theoretical efforts devoted to some important reactions involved in the cold modes of $\mathrm{H}$ burning. As such, it provides some update of more thorough presentations that can be found in e.g. RR88.

\section{THE p-p CHAINS}

The p-p chains, which involve the reactions displayed in Fig. 1, are expected to take place in main sequence $\mathrm{M} \lesssim 1.3 \mathrm{M}_{\odot}$ stars of typical Pop I composition, as well as in more massive stars if their CNO content is low enough. Their net result is the transformation of $\mathrm{H}$ into ${ }^{4} \mathrm{He}$.

Much experimental and theoretical efforts have been devoted to the reactions of the p-p chains, that flurry of activity being of course directly connected with the famed "solar neutrino problem" (e.g. Bahcall 1989; Bahcall and Pinsonneault 1992, hereafter BP92; Ulrich, this conference). The status of our knowledge concerning the pp reactions has already been reviewed in great detail recently (e.g. RR88; Bahcall 1989; BP92), so that we just limit ourselves here to some brief comments.

As no direct measurement of the ${ }^{1} \mathrm{H}\left(\mathrm{p}, \mathrm{e}^{+} \nu\right)^{2} \mathrm{H}$ and ${ }^{1} \mathrm{H}\left(\mathrm{pe}^{-}, \nu\right)^{2} \mathrm{H}$ rates can be expected in any foreseeable future, their knowledge relies entirely on theoretical evaluations. Recent works on that subject have been exhaustively reviewed by BP92 (note that Kurucz (1992) has claimed that the ${ }^{1} \mathrm{H}\left(\mathrm{pe}^{-}, \nu\right){ }^{2} \mathrm{H}$ rate has been incorrectly evaluated in the past. This claim has to be disregarded, as shown in an unpublished note by Bahcall and Salpeter).

A recent reanalysis of the ${ }^{3} \mathrm{He}\left({ }^{3} \mathrm{He}, 2 \mathrm{p}\right){ }^{4} \mathrm{He}$ rate taking into account laboratory electron screening corrections (see below) leads BP92 to adopt an astrophysical S-factor that is $3 \%$ lower than reported by Bahcall (1989). 


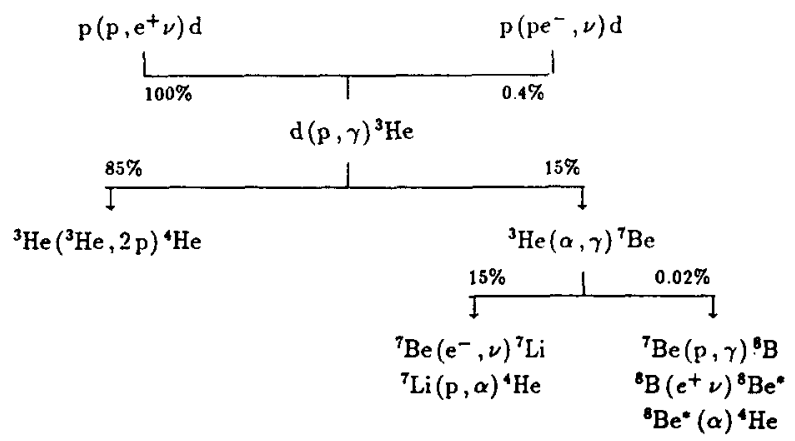

Figure 1. Reactions involved in the pp chains. The indicated percentages give the fractions of the $4 \mathrm{p} \rightarrow \alpha+2 e^{+}+2 \nu$ transformations due to the various possible pp branchings in solar conditions (Bahcall 1989). The very weak $(0.00002 \%)$ ${ }^{3} \mathrm{He}\left(\mathrm{p}, \mathrm{e}^{+} \nu\right){ }^{4} \mathrm{He}$ termination is not indicated

Based on theoretical arguments, it has been suggested that a lowenergy resonance might exist in that reaction (e.g. Fetisov and Kopysov 1975), leading to an enhancement of its rate and a corresponding lowering of the relative importance of the ${ }^{3} \mathrm{He}+{ }^{4} \mathrm{He}$ channel, and thus of the solar ${ }^{8} \mathrm{~B}$ neutrino flux. The latest experimental data on that reaction (Krauss et al. 1987), as well as previous laboratory works, are consistent with a nonresonant reaction mechanism, at least down to $E_{\mathrm{cm}}=24.5 \mathrm{keV}$. They cannot exclude the presence of a narrow $\left(\Gamma_{R}<<24 \mathrm{keV}\right)$ resonance at lower energies. Recent microscopic calculations (Vasilevskii and Rybkin 1989) do not show any sign of such a resonance. Even so, its existence cannot be firmly excluded, and it is of some interest to test its impact on solar models and on the predicted ${ }^{8} \mathrm{~B} \nu$-flux. To this end, evolutionary computations have been performed with the addition to the ${ }^{3} \mathrm{He}\left({ }^{3} \mathrm{He}, 2 \mathrm{p}\right){ }^{4} \mathrm{He}$ non-resonant rate of the contribution of a resonance able to reduce by a factor of 2 the standard ${ }^{8} \mathrm{~B} \nu$-flux while fulfilling the above-mentioned experimental constraints. Those calculations indicate that the required reduction is achieved with a resonance increasing the standard rate by a factor of $\approx 5$. Concomitantly, a $\approx 55 \%$ reduction in the ${ }^{7} \mathrm{Be} \nu$-flux is derived, while the total $\nu$-flux and the other observable solar properties (including the ${ }^{3} \mathrm{He}$ surface abundance) can be recovered.

As far as the remaining ${ }^{3} \mathrm{He}$ burning channels are concerned, the ${ }^{3} \mathrm{He}(\alpha, \gamma){ }^{7} \mathrm{Be}$ rate is estimated to be known to within a $3 \sigma$ error of $6 \%$ (Bahcall 1989), while the recently studied very weak ${ }^{3} \mathrm{He}\left(\mathrm{p}, \mathrm{e}^{+} \nu\right){ }^{4} \mathrm{He}$ branching is considered still to be uncertain by a factor of 6 (BP92). This affects only the corresponding (hep) neutrino flux.

Even if it just represents a weak branching in the pp chains, the ${ }^{7} \mathrm{Be}$ proton capture rate is of hey importance in solar neutrino calculations. 
Unfortunately, there are still significant uncertainties in its low-energy cross section. This is due in particular to the extreme difficulty of performing direct measurements on that reaction (e.g. RR88). In such conditions, indirect methods complemented with model predictions have to be used, this approach having uncertainties of its own. From a critical analysis of those problems, Barker and Spear (1986) propose an astrophysical S-factor for the reaction that is $\approx 30 \%$ lower than the latest model predictions (Johnson et al. 1992) adopted by BP92.

The ${ }^{7} \mathrm{Be}$ electron-capture rate, to which the solar ${ }^{8} \mathrm{~B} \nu$-flux is inversely proportional, has been reexamined in detail by Johnson et al. (1992).

Their proposed slight revision has an unsignificant impact on the $\nu$-flux calculations.

We end this section with some comments on the role of electron screening on nuclear reaction rates at very low energies. Since the pioneering studies of Schatzman (1948) and Salpeter (1954) on stellar electron screening, it is well known that charged-particle induced reactions taking place in a stellar plasma are more rapid than if they were just involving bare nuclei. It has been a common practice to evaluate the stellar rates by multiplying the experimentally derived rates by some correction factor $f_{*}$ that is just slightly in excess of unity (by some percent to some tens of percent) in conditions relevant to main sequence stars.

Painstaking laboratory efforts have made some cracks appear in this whole procedure. Experiments conducted on light species down to energies below $\approx 10 \mathrm{keV}$ indeed reveal an unexpected rise of the astrophysical S-factor with decreasing energy (Engstler et al. 1988; Engstler et al. 1992). This behavior has been assigned to the effect of an electron screening in laboratory conditions, leading to an energy dependent laboratory correction factor $f_{\text {exp }}$. Model predictions do not fully account for the experimentally determined $f_{\text {exp }}$, even if they explain the S-factor rise in a qualitative way (Assenbaum et al. 1987; Bracci et al. 1990, 1991 ; Azrak 1992; Wauters 1992).

At this point, the situation thus appears to be more intricate than previously imagined, a multistep process being required in order to go from laboratory data to stellar rates : (1) the laboratory electron screening effect $\left(f_{\text {exp }}\right)$ has to be extracted from the experimental cross section data in order to get the S-factors for bare nuclei, and (2) the latter factors have to be corrected for the stellar electron screening $\left(f_{*}\right)$ in order to evaluate the stellar rates. This procedure obviously increases the uncertainties that can affect those rates.

\section{THE CNO CYCLES}

The reactions involved in the CNO cycles are represented in Fig. 2. The nuclear physics problems raised by their rate determinations have been discussed in several places (e.g. RR88), so that we limit ourselves here to some brief comments on the nuclear physics and astrophysics aspects of those reaction chains.

The net result of the CNO cycles is the transformation of $\mathrm{H}$ into ${ }^{4} \mathrm{He}$ using the $\mathrm{C}, \mathrm{N}$ and $\mathrm{O}$ isotopes as catalysts. Cycle I (or CN cycle) is the 


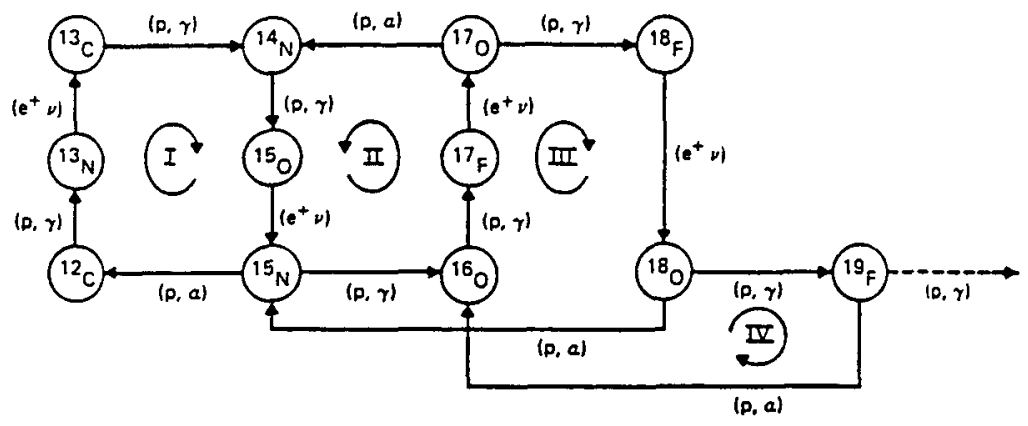

Figure 2. Illustration of the four CNO cycles involved in the transformation of $\mathrm{H}$ into ${ }^{4} \mathrm{He}$. The weak ${ }^{19} \mathrm{~F}(\mathrm{p}, \gamma){ }^{20} \mathrm{Ne}$ leak out of the cycles is also indicated

largest energy producer (e.g. RR88), while all cycles I to IV are important as far as nucleosynthesis is concerned. Figure 3 presents the evolution of the abundances of the $\mathrm{C}, \mathrm{N}, \mathrm{O}$ and $\mathrm{F}$ isotopes as the burning proceeds at a given temperature and density, as well as the final abundances of those isotopes at hydrogen exhaustion versus temperature (assumed to remain constant during the whole burning). It clearly appears that the initial catalysts are mainly transformed into ${ }^{14} \mathrm{~N}$. This well known effect results from the relative slowness of the ${ }^{14} \mathrm{~N}(\mathrm{p}, \gamma){ }^{15} \mathrm{O}$ reaction. It now remains to be seen if those predictions concerning the nucleosynthesis and energy production can be significantly affected by remaining reaction rate uncertainties.

The status of our knowledge concerning the rates of the reactions involved in cycle I of Fig. 2 has been reviewed by RR88, while Bahcall (1989) provides an estimate of the remaining uncertainties.

The reactions of cycles II-IV of Fig. 2 have been discussed in detail by RR88. Since their review, much experimental work has been devoted to the ${ }^{17} \mathrm{O}(\mathrm{p}, \alpha){ }^{14} \mathrm{~N}$ and ${ }^{17} \mathrm{O}(\mathrm{p}, \gamma){ }^{18} \mathrm{~F}$ reactions in order to reduce the very large uncertainties that were still affecting their rates, as reported by e.g. Caughlan and Fowler (1988; hereafter CF88). Those experiments and their results are discussed in detail by Bogaert et al. (1989), Landré et al. (1989) and Berheide et al. (1992). At temperatures of astrophysical interest, it appears that the ${ }^{17} \mathrm{O}(\mathrm{p}, \alpha){ }^{14} \mathrm{~N}$ and ${ }^{17} \mathrm{O}(\mathrm{p}, \gamma){ }^{18} \mathrm{~F}$ rates could be substantially different from the widely used CF88 values. However, uncertainties still remain. They affect in particular the ${ }^{17} \mathrm{O}(\mathrm{p}, \alpha){ }^{14} \mathrm{~N} /{ }^{17} \mathrm{O}(\mathrm{p}, \gamma){ }^{18} \mathrm{~F}$ branching ratio that determines the leakage from cycle II to cycle III. They also have a profound influence on the detailed CNO yield predictions. The standard results shown in Fig. 3 are obtained with values for the ${ }^{17} \mathrm{O}$ p-capture rates that are the geometrical means of the lower and upper limits provided by Landré et al. (1990). The impact of their estimated nuclear uncertainties on the oxygen isotopic composition is displayed in Fig. 4. Also indicated are the results obtained with the upper 


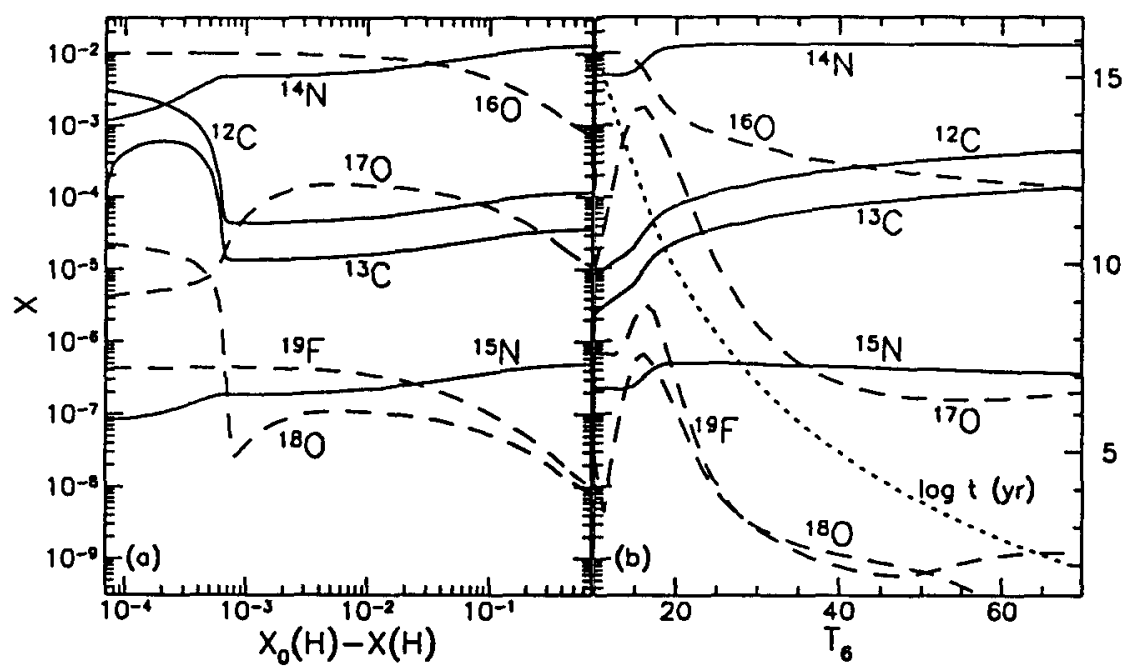

Figure 3. (a) Time evolution of the mass fractions of the stable nuclides involved in the CNO cycles versus the amount of hydrogen burned at constant temperature $\mathrm{T}=25 \times 10^{6} \mathrm{~K}$ and density $\rho=$ $100 \mathrm{~g} / \mathrm{cm}^{3} \cdot \mathrm{X}_{0}(\mathrm{H})=0.7$ is the initial $\mathrm{H}$ mass fraction; (b) same as (a), but versus $\mathrm{T}_{6}$ (temperature in $10^{6} \mathrm{~K}$ ) at $\mathrm{H}$ exhaustion $(\mathrm{X}(\mathrm{H})$ $\left.=10^{-9}\right)$. The short-dashed line indicates the logarithm of the Hburning time (in yr) to be read on the right-hand side ordinate

limits of the ${ }^{17} \mathrm{O}$ p-capture rates derived by Berheide et al. (1992), those upper limits being inferior to the lower bounds proposed by Landré et al. (1990). The impact of the revised ${ }^{17} \mathrm{O}$ p-capture rates has also been studied in the framework of more realistic stellar models by Landré et al. (1990).

The ${ }^{18} \mathrm{O}$ burning has been studied recently by Champagne and Pitt (1986) and Berheide et al. (1992). It proceeds predominantly through ${ }^{18} \mathrm{O}(\mathrm{p}, \alpha){ }^{15} \mathrm{~N},{ }^{18} \mathrm{O}(\mathrm{p}, \gamma){ }^{19} \mathrm{~F}$ being roughly 200 times slower at relevant temperatures.

Recently, the ${ }^{19} \mathrm{~F}$ proton capture rates have been studied experimentally and theoretically (Kious 1990 ; Bulski 1992, and references therein). Even if uncertainties remain in the astrophysical rates, it can be concluded that ${ }^{19} \mathrm{~F}(\mathrm{p}, \alpha){ }^{16} \mathrm{O}$ is much faster than ${ }^{19} \mathrm{~F}(\mathrm{p}, \gamma){ }^{20} \mathrm{Ne}$, preventing any important leakage out of the CNO cycles to ${ }^{20} \mathrm{Ne}$. In addition, the $(p, \alpha)$ channel is so rapid that the ${ }^{19} \mathrm{~F}$ amount that can emerge from cycle IV is too low for accounting for the solar system ${ }^{19} \mathrm{~F}$, in spite of its relatively very low abundance (Goriely et al. 1989; Kious 1990). In the same way, the 

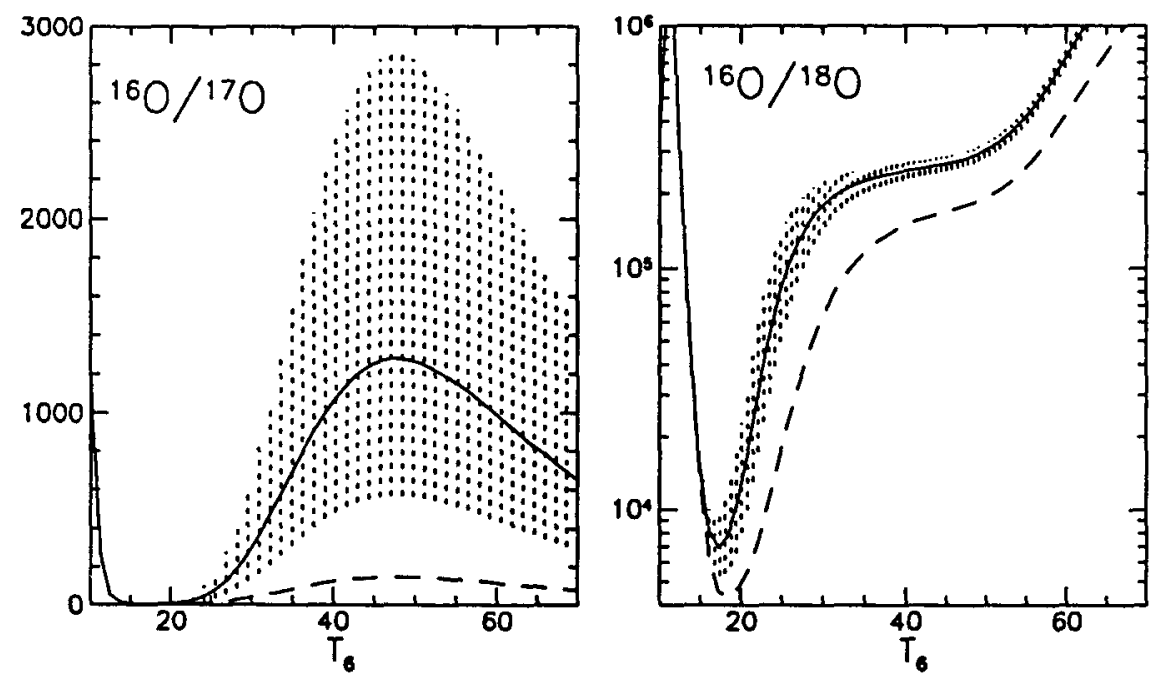

Figure 4. ${ }^{16} \mathrm{O} /{ }^{17} \mathrm{O}$ and ${ }^{16} \mathrm{O} /{ }^{18} \mathrm{O}$ abundance ratios versus (constant) temperature (in $10^{6} \mathrm{~K}$ ) at $\mathrm{H}$ exhaustion for various values of the ${ }^{17} \mathrm{O}(\mathrm{p}, \alpha){ }^{14} \mathrm{~N}$ and ${ }^{17} \mathrm{O}(\mathrm{p}, \gamma){ }^{18} \mathrm{~F}$ rates. The solid and dashed lines are obtained with the intermediate rates used in Fig. 3. The shaded areas indicate the impact of the remaining nuclear uncertainties clajmed by Landré et al. (1990). The dashed lines result from the use of the upper limit put by Berheide et al. (1992) on the ${ }^{17} \mathrm{O} p$ capture rates

CNO cycles cannot account for the ${ }^{19} \mathrm{~F}$ recently observed in Asymptotic Giant Branch stars (Jorissen et al. 1992).

\section{THE NeNa CYCLE}

The nuclear information of relevance for that cycle, represented in Fig. 5, is reviewed by RR88. Let us just stress that still large uncertainties remain in the rates of many of the involved proton captures (see also the analysis of the status of the ${ }^{20} \mathrm{Ne}(\mathrm{p}, \gamma){ }^{21} \mathrm{Na}$ reaction by Prantzos et al. 1991). Those uncertainties cannot affect the conclusion that the ${ }^{23} \mathrm{Na}(\mathrm{p}, \alpha)^{20} \mathrm{Ne}$ reaction rate is large enough compared to the competing ${ }^{23} \mathrm{Na}(\mathrm{p}, \gamma){ }^{24} \mathrm{Mg}$ channel to guarantee cycling.

Figure 6 presents the $\mathrm{NeNa}$ cycle yield predictions derived with the CF88 rates. When their expressions contain an uncertainty factor $F$, "intermediate" rate values $(F=0.1)$ are selected. Upper $(F=1)$ and lower $(\mathrm{F}=0)$ limits to the rates involved in the production and destruction of ${ }^{23} \mathrm{Na}$ 


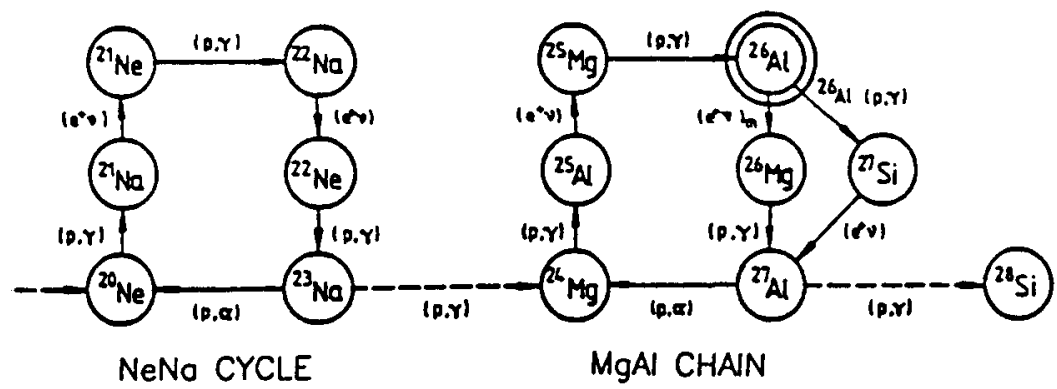

Figure 5. Reactions involved in the $\mathrm{NeNa}$ cycle and in the $\mathrm{MgAl}$ chain. The leakages into and out of those reaction sequences are also schematized. The ground $\left({ }^{26} \mathrm{Al}^{\mathrm{g}} ; t_{1 / 2}=7.05 \times 10^{5} \mathrm{yr}\right)$ and isomeric $\left({ }^{26} \mathrm{Al}{ }^{\mathrm{m}} ; t_{1 / 2}=6.3 \mathrm{~s}\right)$ states of ${ }^{26} \mathrm{Al}$ are considered as two separate species, as they cannot reach thermal equilibrium in realistic conditions of operation of the $\mathrm{MgAl}$ chain (Nørgaard 1979; Ward and Fowler 1980).

are also used to evaluate the impact of the nuclear uncertainties on the ${ }^{22} \mathrm{Ne}$ and ${ }^{23} \mathrm{Na}$ yields. It is seen that only modest abundance spreads result.

The nucleosynthesis associated with the $\mathrm{NeNa}$ cycle has been computed in the framework of detailed stellar models, and its impact on the surface composition of Wolf-Rayet or of Red Giant stars has been analyzed (e.g. Prantzos et al. 1986, 1991). Let us note in particular that the $\mathrm{Na}$ excess observed in certain yellow supergiants (e.g. Boyarchuk et al. 1989) and seemingly correlated with the ${ }^{12} \mathrm{C} /{ }^{13} \mathrm{C}$ ratio has been interpreted in the framework of the $\mathrm{NeNa}$ cycle. However, that interpretation has a chance to account successfully for the observations only if some special assumptions are made on the initial abundance of ${ }^{22} \mathrm{Ne}$ (e.g. Denisenkov 1990), or if the ${ }^{20} \mathrm{Ne}(\mathrm{p}, \gamma){ }^{21} \mathrm{Na}$ rate is artificially enhanced by a factor of at least $\approx 10$ over its currently adopted CF88 value (Prantzos et al. 1991). Both proposed solutions appear quite unlikely. It remains that the observed $\mathrm{Na}$ excess presents a new and interesting challenge for theorists of stellar evolution and nucleosynthesis.

\section{THE MgAl CHAIN}

Much nuclear physics effort has been devoted to the reactions of the $\mathrm{MgAl}$ chain displayed in Fig. 5, and in particular to those involved in the production or destruction of ${ }^{26} \mathrm{Al}$ and ${ }^{27} \mathrm{Al}$. This directly relates to the recognition that ${ }^{26} \mathrm{Al}{ }^{8}$ is of crucial importance in $\gamma$-ray astronomy and in cosmochemistry $\left({ }^{26} \mathrm{Al}^{\mathrm{m}}\right.$ is so short-lived that it is of no practical interest in 


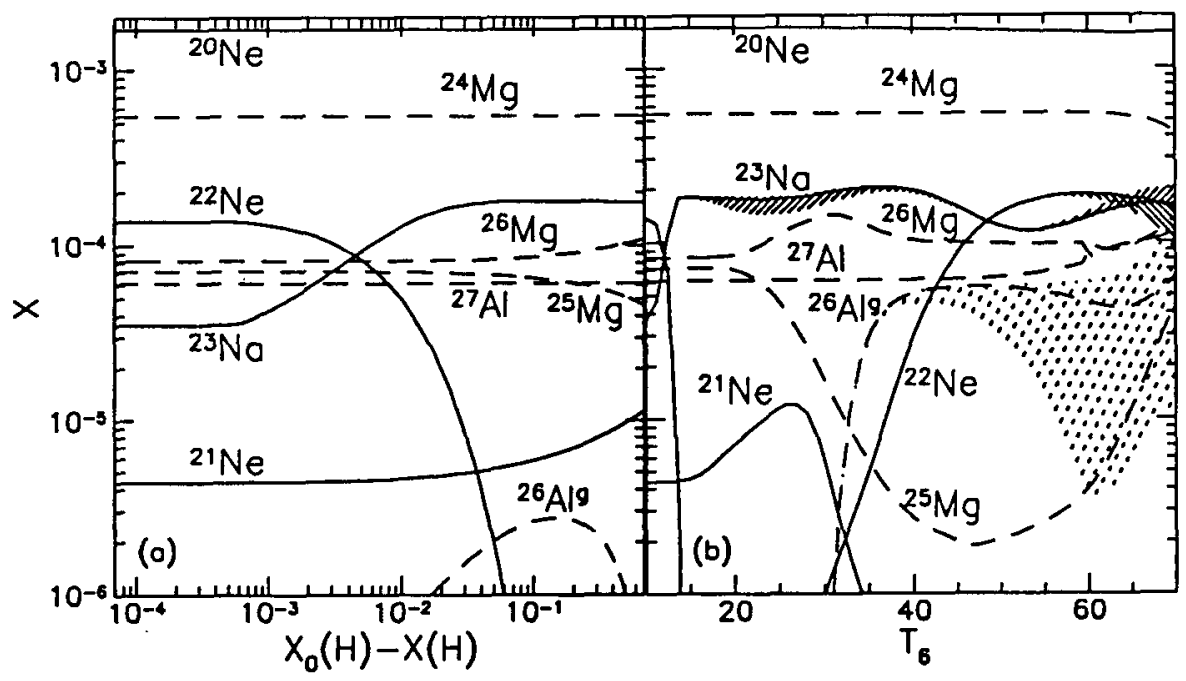

Figure 6. Same as Fig. 3, but for the nuclides involved in the $\mathrm{NeNa}$ cycle (solid lines) and $\mathrm{MgAl}$ chain (dashed lines). Data for ${ }^{26} \mathrm{Al}^{8}$ are also presented. The hatched zones indicate the ${ }^{22} \mathrm{Ne},{ }^{23} \mathrm{Na}$ or ${ }^{26} \mathrm{Al}{ }^{8}$ abundance uncertainties of nuclear origin (see text and Fig. 7)

those respects). On the one hand, the $1.8 \mathrm{MeV} \gamma$-ray emission observed in the galactic disk is attributed to the decay of $\approx 3 \mathrm{M}_{\odot}$ of ${ }^{26} \mathrm{Al}^{\mathrm{g}}$ that have been present in the interstellar medium over the last $\approx 10^{6} \mathrm{yr}$ (e.g. Schönfelder and Varendorff 1991; Clayton and Leising 1987 for reviews). On the other hand, there is now ample observational evidence that ${ }^{26} \mathrm{Al}{ }^{8}$ has decayed in situ in various meteoritic inclusions, as well as in identified single grains of likely stellar origin (e.g. Wasserburg 1985; Zinner et al. 1991; Amari et al. 1992).

Recently, the rate of the ${ }^{25} \mathrm{Mg}$ proton captures leading to both ${ }^{26} \mathrm{Alg}$ and ${ }^{26} \mathrm{Al}^{\mathrm{m}}$ has been studied experimentally by liadis et al. (1990), and is now put on quite safe grounds. The derived results are significantly different (factor $\gtrsim 5$ ) from the previously adopted CF88 ones for temperatures $T$ $\lesssim 10^{8} \mathrm{~K}$. The branching ratio for forming ${ }^{26} \mathrm{Al}$ through ${ }^{25} \mathrm{Mg}$ p-capture has received much experimental attention as well (Endt and Rolfs 1987). The ${ }^{26} \mathrm{Al}^{8}(\mathrm{p}, \gamma){ }^{27} \mathrm{Si}$ reaction has also been studied recently (Vogelaar 1989). The deduced rate appears to be lower than the ${ }^{26} \mathrm{Alg} \beta$-decay rate for $\mathrm{T} \lesssim$ $3-4 \times 10^{8} \mathrm{~K}$. In other words, ${ }^{26} \mathrm{Al}$ is predominantly destroyed by $\beta$-decay in those conditions, and by ${ }^{26} \mathrm{Al}^{\mathrm{g}}(\mathrm{p}, \gamma)^{27} \mathrm{Si}$ if the $\mathrm{MgAl}$ chain can indeed take place at higher temperatures, which may not be the case in realistic astrophysical situations. If indeed the ${ }^{26} \mathrm{Alg}$ p-capture channel is significant, the uncertainties in its rate influence the reliability of the ${ }^{26} \mathrm{Al} 1^{8}$ yield predictions. In addition ${ }^{26} \mathrm{Al}^{\mathrm{g}}$ is connected electromagnetically to ${ }^{26} \mathrm{Al}^{\mathrm{m}}$. 
The $\gamma$-ray transitions of relevance in astrophysical conditions have been discussed recently (Vogelaar 1989). The ${ }^{26} \mathrm{Al}^{\mathrm{m}}$ isomer can also transform by ${ }^{26} \mathrm{Al}^{\mathrm{m}}(\mathrm{p}, \gamma){ }^{27} \mathrm{Si}$. Due to the very short ${ }^{26} \mathrm{Al}^{\mathrm{m}} \beta$-decay half-life, that reaction has not been amenable yet to any experimental study, the evaluation of its rate thus relying on theoretical evaluations only (e.g. Arnould et al. 1980). Recent experiments have also been carried out on the ${ }^{26} \mathrm{Mg}$ and ${ }^{27} \mathrm{Al}$ proton capture rates. The new data obtained for ${ }^{26} \mathrm{Mg}(\mathrm{p}, \gamma){ }^{27} \mathrm{Al}$ (Iliadis et al. 1990) leave uncertainties that are less than a factor of $\approx 2$ for $\mathrm{T} \lesssim 3 \times 10^{7}$ $\mathrm{K}$, but that can reach factors of $\approx 10$ to 100 in the $4 \times 10^{7} \lesssim T \lesssim 6 \times 10^{7} \mathrm{~K}$ range. As already stressed before, these temperatures may be a bit high for the operation of the cold $\mathrm{MgAl}$ chain in realistic astrophysical situations, so that the corresponding large uncertainties may have only little astrophysical consequences in practice.

From their experimental data concerning ${ }^{27} \mathrm{Al}(\mathrm{p}, \gamma){ }^{28} \mathrm{Si}$, lliadis et al. (1990) suggest to use the CF88 rate. In contrast, the CF88 rate for ${ }^{27} \mathrm{Al}(\mathrm{p}, \alpha){ }^{24} \mathrm{Mg}$ is subject to important changes, following the laboratory work of Champagne et al. (1988) and Timmermann et al. (1988). From these recent data, it appears in particular that ${ }^{27} \mathrm{Al}(\mathrm{p}, \alpha){ }^{24} \mathrm{Mg}$ may be slower than ${ }^{27} \mathrm{Al}(\mathrm{p}, \gamma){ }^{28} \mathrm{Si}$, so that a $\mathrm{MgAl}$ cycle cannot set up, in contrast to what was thought previously. Further work is required in order to reduce the uncertainties still affecting the ${ }^{27} \mathrm{Al}(\mathrm{p}, \alpha){ }^{24} \mathrm{Mg}$ and ${ }^{27} \mathrm{Al}(\mathrm{p}, \gamma)^{28} \mathrm{Si}$ rates, and thus the precise extent of the leakage out of the $\mathrm{MgAl}$ region.

Figure 6 presents the abundances of the $\mathrm{Mg}$ and $\mathrm{Al}$ isotopes involved in the $\mathrm{MgAl}$ chain. These results are obtained in the framework of a schematic astrophysical model assuming constant temperature and density during hydrogen burning. On the other hand, the CF 88 rates are used, except for the above-mentioned reactions for which more recent data are available. In this case, intermediate rates (geometrical means) are adopted when only upper and lower bounds can be provided experimentally. It appears in particular that a substantial amount of ${ }^{26} \mathrm{~A} 1^{8}$ can be produced at the expense of ${ }^{25} \mathrm{Mg}$ (Fig. 6(a)). However, not much ${ }^{26} \mathrm{Al}^{\mathrm{g}}$ remains at hydrogen depletion for $\mathrm{T} \lesssim 3 \times 10^{7} \mathrm{~K}$ (Fig. 6(b)). This is essentially due to the fact that the synthesized ${ }^{26} \mathrm{Al}{ }^{\mathrm{g}}$ has time to $\beta$-decay before the end of $\mathrm{H}$ burning.

The impact of the uncertainties remaining in the rates of the reactions involved in the production and destruction of ${ }^{26} \mathrm{Al}^{\mathrm{g}}$ and ${ }^{27} \mathrm{Al}$ on the predicted abundances of those nuclides can be evaluated from Figs. 6 and 7 (see also lliadis et al. 1990).

The MgAl chain has also been studied in the framework of detailed stellar models. In particular, the ${ }^{26} \mathrm{Al} \mathrm{l}^{8}$ yields from the core H-burning phase of Wolf-Rayet stars (e.g. Prantzos et al. 1986; Meynet and Arnould 1992), or from the shell $\mathrm{H}$ burning in low- and intermediate- mass thermally pulsing Asymptotic Giant Branch stars (Forestini et al. 1992) have been computed. It is concluded that those sites can be significant contributors to the present day interstellar medium ${ }^{26} \mathrm{Al}$, even if they cannot account for the whole $3 \mathrm{M}_{\odot}$ of ${ }^{26} \mathrm{Al}$ inferred from observation (see also Prantzos 1991). They can also provide a satisfactory explanation for a large variety of ${ }^{26} \mathrm{Al} /{ }^{27} \mathrm{Al}$ ratios that are inferred at the time of formation of inclusions or grains found in meteorites (Meynet and Arnould 1992). 


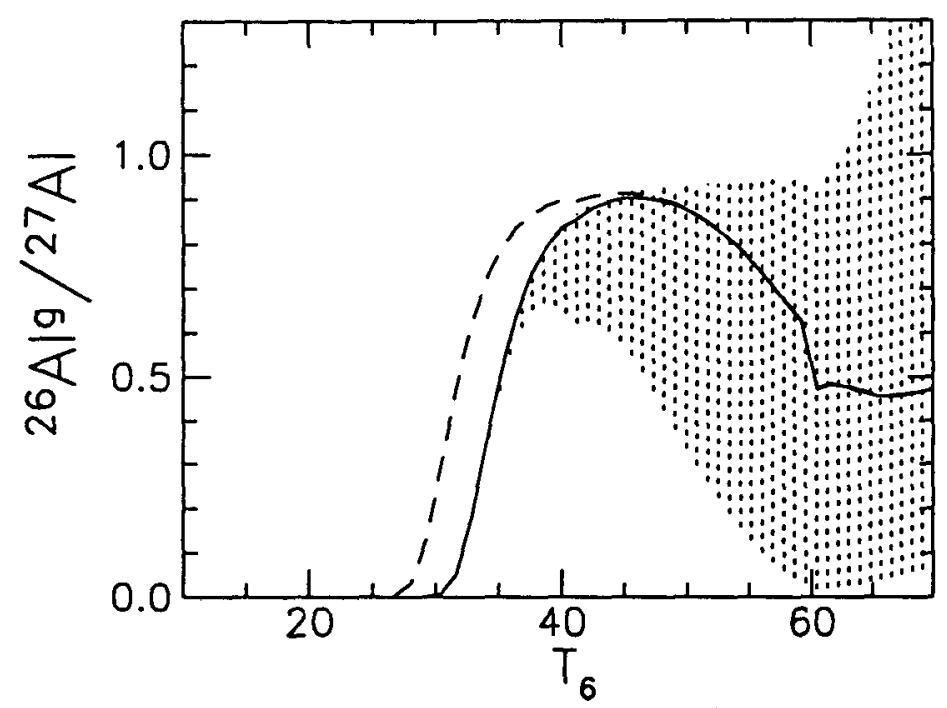

Figure 7. ${ }^{26} \mathrm{Al} /{ }^{87} \mathrm{Al}$ abundance ratio versus (constant) temperature (in $10^{6} \mathrm{~K}$ ) close to $\mathrm{H}$ exhaustion. The solid and dashed lines correspond to final $\mathrm{H}$ mass fractions $\mathrm{X}(\mathrm{H})=10^{-9}$ and $10^{-2}$, respectively. They are obtained with the adoption of intermediate reaction rates, when appropriate (see text). The minimization or maximization of the abundance ratio is obtained by taking lower or upper bounds (as appropriate) on the rates of ${ }^{26} \mathrm{Mg}(\mathrm{p}, \gamma){ }^{27} \mathrm{Al}$ (Iliadis et al. 1990), ${ }^{26} \mathrm{Al}{ }^{8}(\mathrm{p}, \gamma){ }^{27} \mathrm{Si}$ (Vogelaar 1989), ${ }^{27} \mathrm{Al}(\mathrm{p}, \gamma){ }^{28} \mathrm{Si}$ (CF88), and ${ }^{27} \mathrm{Al}(\mathrm{p}, \alpha){ }^{24} \mathrm{Mg}$. The lower bound on the rate of the latter reaction is deduced from the contribution of the $E_{R} \geq 505 \mathrm{keV}$ resonances evaluated by Timmermann et al. (1988). The maximum contributions of the resonances at lower energies reported by Champagne et al. (1988) are added in order to obtain the upper bound on that reaction rate

\section{CONCLUSIONS AND PROSPECTS}

Over the years, heroic laboratory efforts complemented with lengthy calculations have helped greatly improving our knowledge of the rates of a large variety of nuclear reactions of importance in stellar evolution and nucleosynthesis. A substantial fraction of those activities have focussed on the various cold modes of hydrogen burning briefly reviewed here.

In spite of that, nuclear uncertainties still remain, and can blur (in combination, of course, with uncertainties of astrophysical origin) our understanding of various questions of great astrophysical interest, like the 
solar neutrino problem, or the surface $\mathrm{C}, \mathrm{N}$, and $\mathrm{O}$ elemental and isotopic composition of stars having experienced dredge-up episodes. The puzzle raised by the $\mathrm{Na}$ excess observed in yellow supergiants, or the origin of the ${ }^{26} \mathrm{Al}$ amount inferred to exist in the present day interstellar medium or in meteoritic inclusions or grains at the time of their solidification are other exemples of fascinating questions.

In order to reduce the astrophysically important remaining uncertainties of nuclear origin, further theoretical efforts are required, along with the development of improved and new experimental techniques. Because the cross sections drop by many orders of magnitude when the energies decrease from the Coulomb barrier to the energy range of astrophysical interest, the experimental investigations require the availability of high ionbeam currents, of stable and pure targets, as well as of efficient detectors (e.g. RR88).

New accelerators able to deliver higher currents than previously have just been built or are currently tested. On the other hand, windowless gas targets or specially prepared solid targets can provide the required stability and purity. The implantation technique has also been shown rencently to be able to produce isotopically enriched and contaminant-free targets capable of withstanding high beam powers. As far as detectors are concerned, their choice and design require a careful study for each specific experiment. Much progress has been made recently, in particular through the development of new techniques in the detection of neutrons, charged particles, and $\gamma$-rays. Of course, the sensitivity of the best designed detectors is still limited by the cosmic-ray background. Its reduction by nearly three orders of magnitude can be achieved by carrying out experiments deep underground. Such an exciting possiblity is at the center of the LUNA project to be conducted at the Gran Sasso underground laboratory (Arpesella et al. 1991).

Methods for studying astrophysically important reactions through experiments carried on other reactions from which properties of astrophysical interest can be deduced have also proved in the past to be very helpful. They certainly deserve further developments. Exemples of such indirect techniques include the measurement of inverse reactions, or the Coulomb dissociation approach.

All those dedicated nuclear physics efforts will undoubtedly bring much progress in our knowledge of the stellar nuclear "alchemy". In particular, they will help reducing possible ambiguities in the interpretation of solar neutrino experiments (Bahcall 1989).

Aknowledgements. This work has been supported in part by the SCIENCE Program SC1-0065. M.A. is Chercheur Qualifié F.N.R.S. (Belgium), and N.M. is Boursier I.R.S.I.A.

\section{REFERENCES}

Amari, S., Hoppe, P., Zinner, E. and Lewis, R. 1992, Lunar Planetary Science XXIII, to appear

Arnould, M., Nørgaard, H., Thielemann, F.K. and Hillebrandt, W. 1980, Astrophys. J., 237, 931 
Arpesella, C. et al. 1991, LNGS Report 91/18

Assenbaum, H.J., Langanke, K. and Rolfs, C. 1987, Z. Phys., A327, 461

Azrak, Z. 1992, Ph.D. Thesis, Université Libre de Bruxelles

(unpublished)

Bahcall, J.N 1989, Neutrino Astrophysics (Cambridge University Press, Cambridge)

Bahcall, J.N. and Pinsonneault, M.H. 1992, Astrophysics Preprint Series IASSNS-AST 92/10 (to be published in Rev. Mod. Phys.) (BP 92)

Barker, F.C. and Spear, R.H. 1986, Astrophys. J., 307, 847

Berheide, M., Rolfs, C., Schröder, U. and Trautvetter, H.P. 1991, preprint (Bochum)

Bethe, H.A. 1939, Phys. Rev., 55, 103 and 434

Bethe, H.A. and Critchfield, C.L. 1938, Phys. Rev., 54, 248 and 862

Bogaert, G.L., Landré, V., Aguer, P., Barhoumi, S., Kious, M., Lefebvre, A., Thibaud, J.-P. and Bertault, D. 1989, Phys. Rev., C39, 1972

Boyarchuk, A.A., Gubeny, I., Kubat, I., Lyubimkov, L.S. and Sakhibullin, N.A. 1989, Astrophysics, 28-29, 197

Bracci, L., Fiorentini, G., Melerzhik, V.S., Mezzorani, G. and Quarati, P. 1990, Nucl. Phys., A513, 316

Bracci, L., Fiorentini, G., Melerzhik, V.S., Mezzorani, G. and Pasini, P. 1991, Phys. Lett., A152, 456

Bulski, G. 1992, Ph.D. Thesis, Universität Stuttgart (unpublished)

Caughlan, G.R. and Fowler, W.A. 1988, Atomic Data Nucl. Data Tables, 40, 283 (CF88)

Champagne, A.E. and Pitt, M.L. 1986, Nucl. Phys., A457, 367

Champagne, A.E., Cella, C.H., Kouzes, R.T., Lowry, M.M., Magnus, P.V.,

Smith, M.S. and Mao, Z.Q. 1988, Nucl. Phys., A487, 433

Clayton, D.D. and Leising, M.D. 1987, Phys. Rep., 144, 1

Denisenkov, P.A. 1990, Astrophysics, 31, 588

Endt, P.M. and Rolfs, C. 1987, Nucl. Phys., A467, 261

Engstler, S. Krauss, A., Neldner, K., Rolfs, C., Schröder, U. and Langanke, K. 1988, Phys. Lett., B202, 179

Engstler, S., Raimann, G., Angulo, G., Greife, U., Rolfs, C., Schröder, U., Somorjai, E., Kirch, B. and Langanke, K. 1992, Phys. Lett., B279, 20, and $Z$. Phys.,A342, 471

Fetisov, V.N. and Kopysov, Y.S. 1975, Nucl. Phys., A239, 511

Forestini, M., Paulus, G. and Arnould, M. 1991, Astron. Astrophys., 252, 597

Forestini, M., Goriely, S., Jorissen, A. and Arnould, M. 1992, Astron. Astrophys., 261, 157

Goriely, S., Jorissen, A. and Arnould, M. 1989, in Proceedings of the 5th Workshop on Nuclear Astrophysics, eds. W. Hillebrandt and E. Müller, MPA/P1 (Max-Planck-Institut für Astrophysik, Garching), p. 60

Iben, I.Jr. 1991, in Evolution of Stars : The Photospheric Abundance Connection, IAU Symposium 145 (eds. G. Michaud and A. Tutukov (Kluwer Academic Pub., Dordrecht), p. 257

liadis, Ch., Schange, Th., Rolfs, C., Schröder, U., Somorjai, E., Trautvetter, H.P., Wolke, K., Endt, P.M., Kikstra, S.W., Champagne, A.E., Arnould, M. and Paulus, G. 1990, Nucl. Phys., A512, 509 
Johnson, C.W., Kolbe, E., Koonin, S.E. and Langanke, K. 1992, preprint

Jorissen, A., Smith, V.V. and Lambert, D.L. 1992, Astron. Astrophys., 261, 164

Kious, M. 1990, Ph. D. Thesis, C.S.N.S.M. Orsay (unpublished)

Krauss, A., Becker, H.W., Trautvetter, H.P. and Rolfs, C. 1987, Nucl. Phys., A467, 273

Kurucz, R.L. 1992, Harvard-Smithsonian Center for Astrophysics preprint 3360

Landré, V., Prantzos, N., Aguer, P., Bogaert, G., Lefebvre, A. and Thibaud, J.-P. 1990, Astron. Astrophys., 240, 85

Landré, V., Aguer, P., Bogaert, G., Lefebvre, A., Thibaud, J.-P., Fortier, S., Maison, J.-M. and Vernotte, J. 1989, Phys. Rev., C40, 1972

Meynet, G. and Arnould, M. 1992, Proceedings of the International Symposium "Origin and Evolution of the Elements", Paris, 22 25 June 1992, to appear

Nørgaard, H. 1979, in Gross Properties of Nuclei and Nuclear Excitations VII (Hirschegg), ed. H. von Groote (Technische Hochschule, Darmstadt), p. 204

Prantzos, N. 1991, in Gamma-ray Line Astrophysics, eds. Ph. Durouchoux and N. Prantzos (A.I.P., New York), p. 129

Prantzos, N., Coc, A. and Thibaud, J.-P. 1991, Astrophys. J., 379, 729

Prantzos, N., Doom, C., Arnould, M. and de Loore, C. 1986, Astrophys. J., 304, 695

Rolfs, C.E. and Rodney, W.S. 1988, Cauldrons in the Cosmos (The University of Chicago Press, Chicago) (RR88)

Salpeter, E.E. 1954, Aust. J. of Phys., 7, 373

Schatzman, E. 1948, J. Phys. Radium, 9, 46

Schönfelder, V, and Varendorff, M. 1991, in Gamma-ray Line Astrophysics, eds. Ph. Durouchoux and N. Prantzos (A.I.P., New York), p. 101

Sneden, C. 1991, Evolution of Stars : The Photospheric Abundance Connection, IAU Symposium 145, eds. G. Michaud and A. Tutukov (Kluwer Academic Pub., Dordrecht), p. 235

Timmermann, R., Becker, H.W., Rolfs, C., Schröder, U. and Trautvetter, H.P. 1988, Nucl. Phys., A477, 105

Vasilevskii, V.S. and Rybkin, I.Yu. 1989, Sov. J. Nucl. Phys., 50, 411

Vogelaar, B. 1989, Ph.D. Thesis, California Institute of Technology (unpublished)

von Weizsäcker, C.F. 1937, Phys. Z., 38, 176

von Weizsäcker, C.F. 1938, Phys. Z., 39, 633

Ward, R.A. and Fowler, W.A. 1980, Astrophys. J., 238, 266

Wasserburg, G.J. 1985, in Protostars and Planets II, eds. D.C. Black and M.S. Matthews ((University of Arizona Press, Tucson), p. 703

Wauters, L. 1992, Master Thesis, Université Libre de Bruxelles (unpublished)

Zinner, E., Amari, S., Anders, E. and Lewis, R. 1991, Nature, 349, 51 